\title{
LA CADENA DE FINANCIACIÓN: UnA NeCESIDAd PARA EL DESARROLLO ECONÓMICO Y SOCIAL A PARTIR DEL EMPRENDIMIENTO
}

\section{Resumen}

Diferentes estudios han planteado insistentemente como una de las principales problemáticas asociadas al desarrollo empresarial, la falta de mecanismos especializados para la financiación tanto de las nuevas empresas, como del desarrollo de las existentes; este artículo plantea claramente las razones que determinan esta afirmación, así como las principales alternativas existentes a nivel mundial para la solución de la problemática, a manera de estado del arte.

Finalmente con la información anteriormente relacionada se plantean entonces una serie de reflexiones y conclusiones alrededor del caso colombiano, por parte de los autores.

\section{Palabras clave}

Financiación

Capital de riesgo

Ángeles inversionistas,

Start Up

Emprendimiento
Francisco Javier Matíz B* Yida Mogollón Cuevas**

\section{INTRODUCCIÓN}

\section{D}

esde hace ya varias décadas se ha llegado a un consenso mundial referente a la clara y directa relación existente entre el desarrollo económico y social de una nación y su capacidad de crecimiento y generación de tejido empresarial a través de la creación de empresas; estudios como el Global Entrepreneurship Monitor ${ }^{1}$ han evidenciado entonces a través de la medición de la Tasa de Actividad Emprendedora la capacidad de los diferentes países de generar nuevas empresas, así como el desarrollo de la industria de soporte necesaria para que estas nuevas unidades productivas

\footnotetext{
* Francisco Javier Matiz B. M.Sc. Magíster en Gestión de Organización, Especialista en Psicología del Consumidor y Profesional en Comercio Internacional; Actualmente se desempeña como Director del Programa Emprendedor y del Grupo de Investigación Entrepreneurship Group de la Universidad EAN.

**Yida Mogollón Cuevas. Especialista en Gerencia de Mercadeo, Administradora de Negocios Internacionales, Investigadora asociada al Entrepreneurship Group de la Universidad EAN.

${ }^{1}$ El Global Entrepreneurship Monitor,es un estudio global de emprendimiento liderado por las universidades Babson Collage y London Business School; en su versión del año 2006 tuvo una cobertura de 42 países a nivel mundial.
}

Este artículo fué entregado el 7 de mayo de 2008 y su publicación aprobada por el Comité Editorial el 18 de junio de 2008. 
puedan ser exitosas y perdurar en el tiempo; ya que son las empresas originadas a partir de oportunidades de mercado, con algunos niveles de innovación y valor agregado aquellas que dinamizan su entorno no solo a través de la generación de empleo, sino también desde su aporte a la calidad de vida de sus usuarios y el incremento de la competitividad de los sectores económicos en que estén inmersas.

Ahora bien, dentro de las principales variables de entorno que estudios como el GEM evalúan a través de un ejercicio de investigación por medio de entrevistas a expertos, tenemos la favorabilidad existente para la creación de empresas y la labor del emprendedor donde se consulta sobre el nivel de desarrollo de criterios tales como la existencia de políticas y programas gubernamentales de apoyo, la capacidad de transferencia tecnológica y de procesos de investigación y desarrollo, la apertura del mercado, las condiciones de la infraestructura física y comercial, las condiciones de educación y capacitación, y el desarrollo del mercado financiero; siendo este último el de menor desempeño para el caso Colombiano, de acuerdo con el informe GEM 2006.

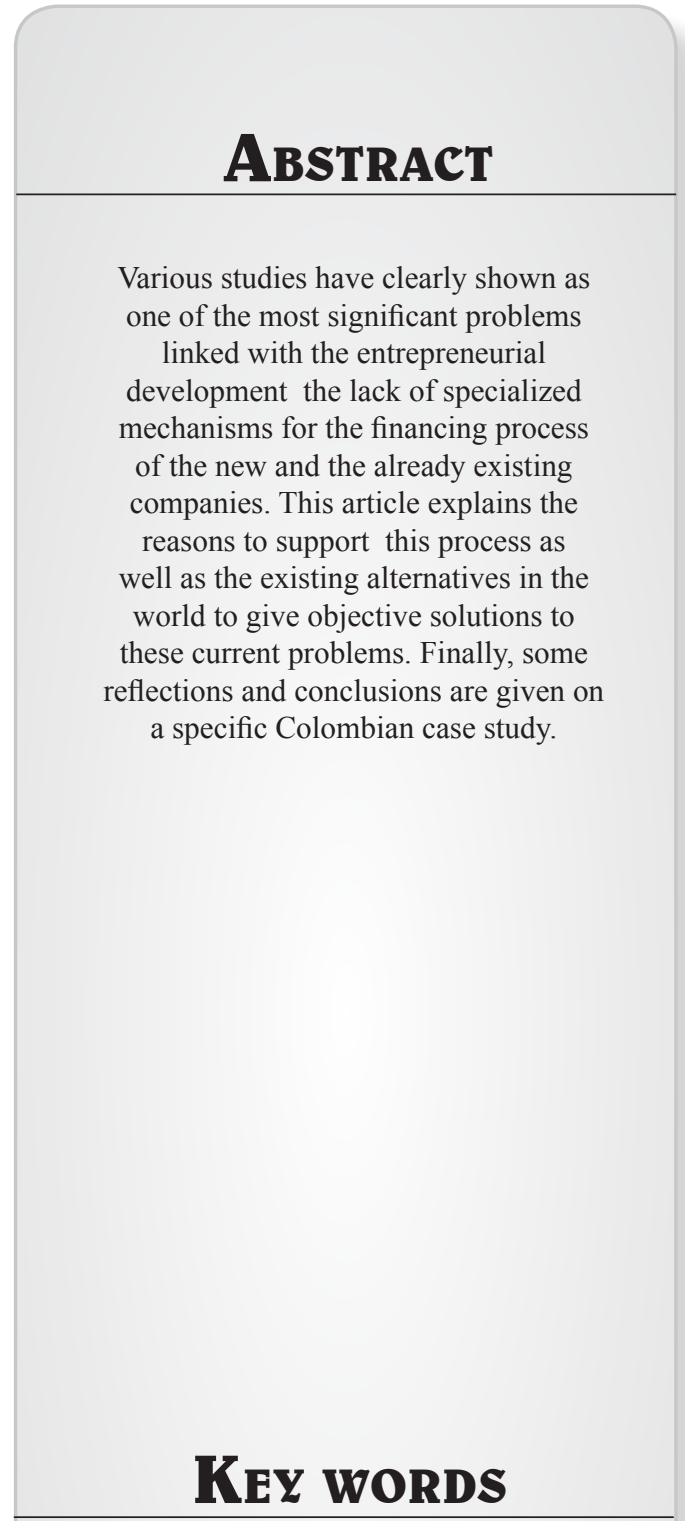

\footnotetext{
Financing Risk capital Investing Angels Start Up Entrepreneurship
} 


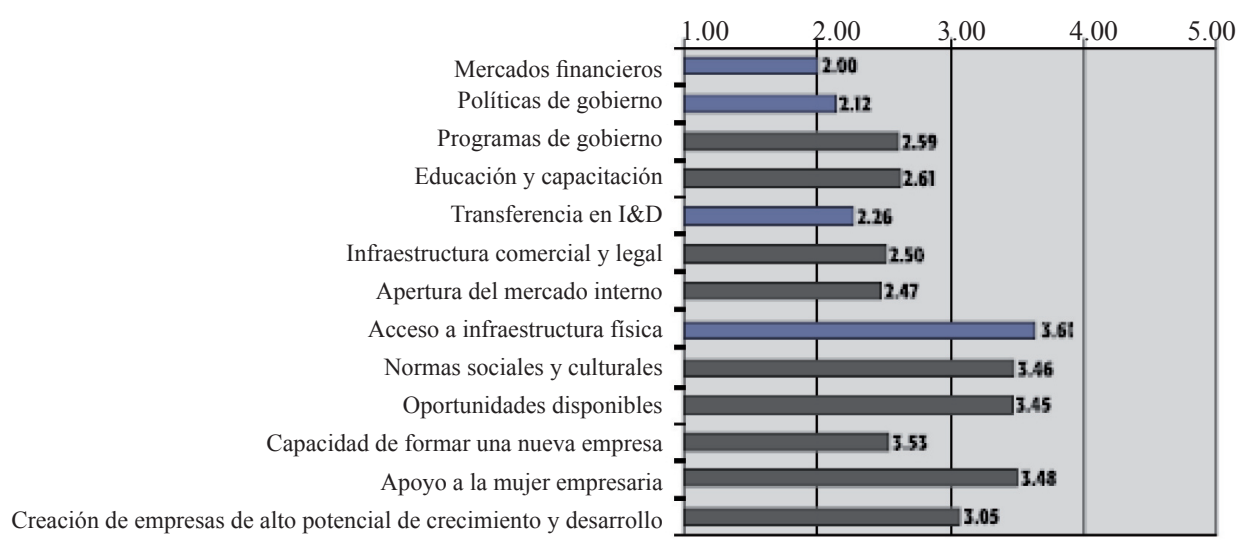

Fuente. Informe GEM Colombia 2006

Referente a esta problemática el estudio menciona que: "En relación con el acceso a los mercados financieros, la opinión de los expertos es que en Colombia los nuevos empresarios prácticamente tienen que depender de sus propios recursos o de los de su entorno (familia, socios, amigos), pues las otras fuentes de financiación son casi inexistentes. Las calificaciones más bajas se dieron a: capital de riesgo, créditos, subsidios del Gobierno, mercado financiero abierto y otras fuentes privadas" ${ }^{2}$, haciendo evidente la problemática a que se enfrentan diariamente los emprendedores y empresarios no solo de Colombia sino de Latinoamérica en general.

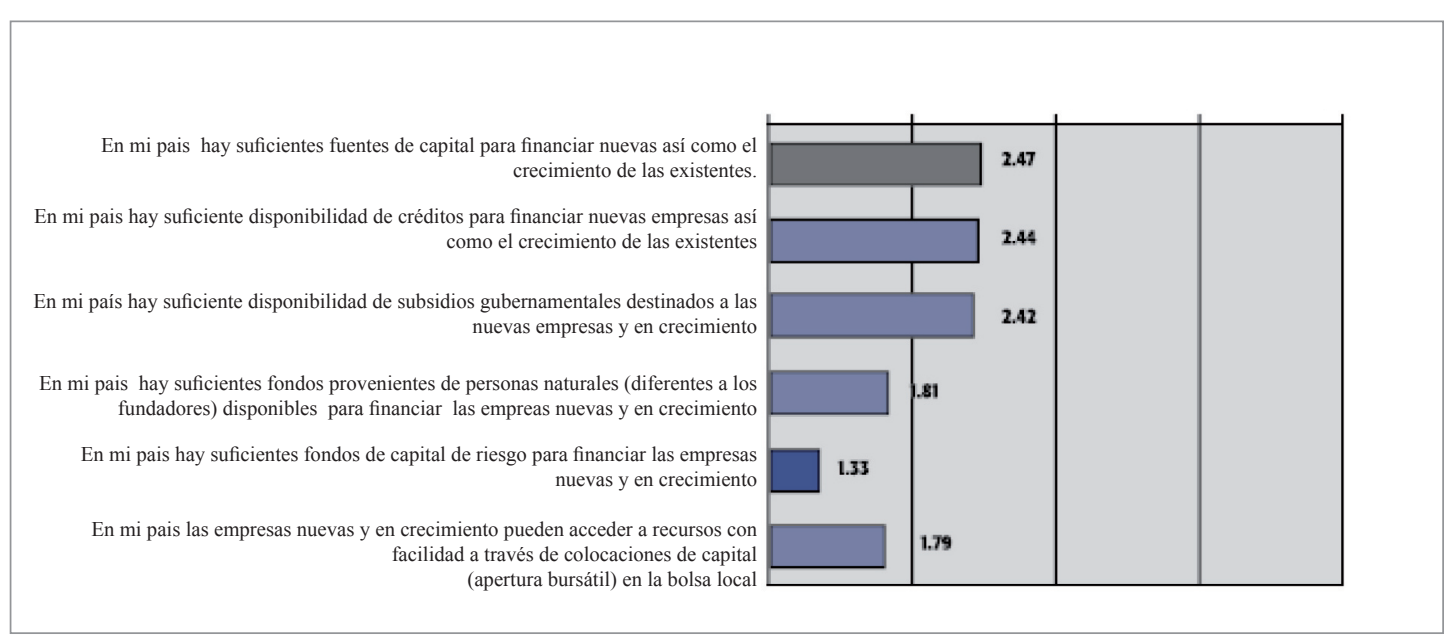

Fuente. Informe GEM Colombia 2006

\footnotetext{
${ }^{2}$ Informe GEM Colombia 2006.
} 
La percepción de la problemática planteada por el GEM 2006 para el caso colombiano, es reafirmada por documentos de política tales como el CONPES 3484 de agosto de 2007 denominado "Política nacional para la transformación productiva y la promoción de las micro, pequeñas y medianas empresas: un esfuerzopúblicoprivado"3.

Este documento presenta cifras alarmantes frente a la problemática de financiación de las empresas en el mercado colombiano, tales como que el $88 \%$ de las microempresas del país son financiadas a través de los limitados recursos de sus fundadores, provenientes de su baja capacidad de ahorro y la de sus familias; esto en gran medida debido a que las características de las microempresas, y en el caso de los emprendimientos de sus gestores, no permiten cumplir con los requisitos planteados por el mercado financiero para acceder a la financiación a través de créditos formales que a su vez de acuerdo con sus características técnicas (Tasas de interés, garantías, plazos, etc.) se hacen igualmente inadecuados para el fortalecimiento del tejido empresarial del país.

Partiendo de esta problemática la Universidad EAN a través de su grupo de Investigación Entrepreneurship Group, a lo largo del año 2007 adelantó el proyecto de investigación "Fuentes de financiación para el Start Up de una empresa"4, con el ánimo de identificar las buenas prácticas en el proceso de financiación empresarial, y específicamente en la creación y puesta en marcha de empresas.

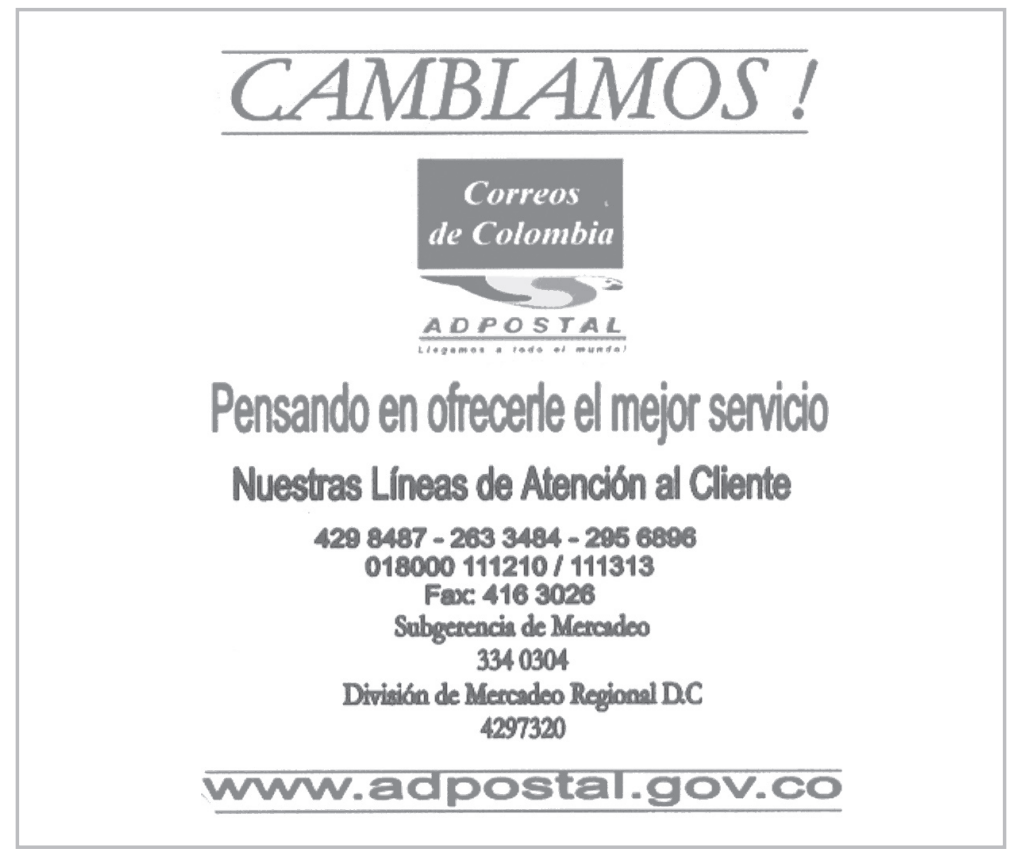

\footnotetext{
${ }^{3}$ Conpes 3484 Política Nacional para la transformación productiva y la promoción de las micro, pequeñas y medianas empresas: Un esfuerzo público-privado. Ministerio de Comercio Industria y Turismo, Departamento Nacional de Planeación - Agosto de 2007.

${ }^{4}$ MOGOLLÓN,Yida. Fuentes de financiación para el Start Up de una empresa. 2007.
} 


\section{Etapas del ciclo de desarrollo de la empresa}

In la actualidad podría decirse que no existe una clasificación única para determinar la duración de las etapas dentro del ciclo de desarrollo de una empresa, básicamente debido a que la evolución de las mismas en el tiempo, depende de una gran variedad de factores tales como el sector económico en que se encuentran, el mercado al cual están dirigidas y las características propias de la organización; encontramos entonces que sin importar el tiempo en que se desarrollen las empresas afrontan básicamente cuatro estadios o etapas dentro de su proceso de desarrollo:

- Planeación o conceptualización

- Start Up

- Crecimiento

- Consolidación
Ahora bien la clasificación de las empresas con relación a su etapa de desarrollo o nivel de crecimiento, conlleva igualmente a problemáticas específicas en cuanto a aspectos tales como su nivel de desarrollo en procesos de investigación y desarrollo, capacidad degeneración denuevos productos o servicios, inserción en los mercados tanto nacionales como internacionales, y obviamente sus necesidades financieras.

Es así como Berger y Udell (1998) sostienen que las PyMEs que tienen una estrategia de crecimiento recorren un ciclo de financiamiento accediendo a diferentes fuentes e instrumentos a medida que la empresa crece, gana experiencia y se hace más transparente. En el inicio de sus actividades, las empresas acceden a

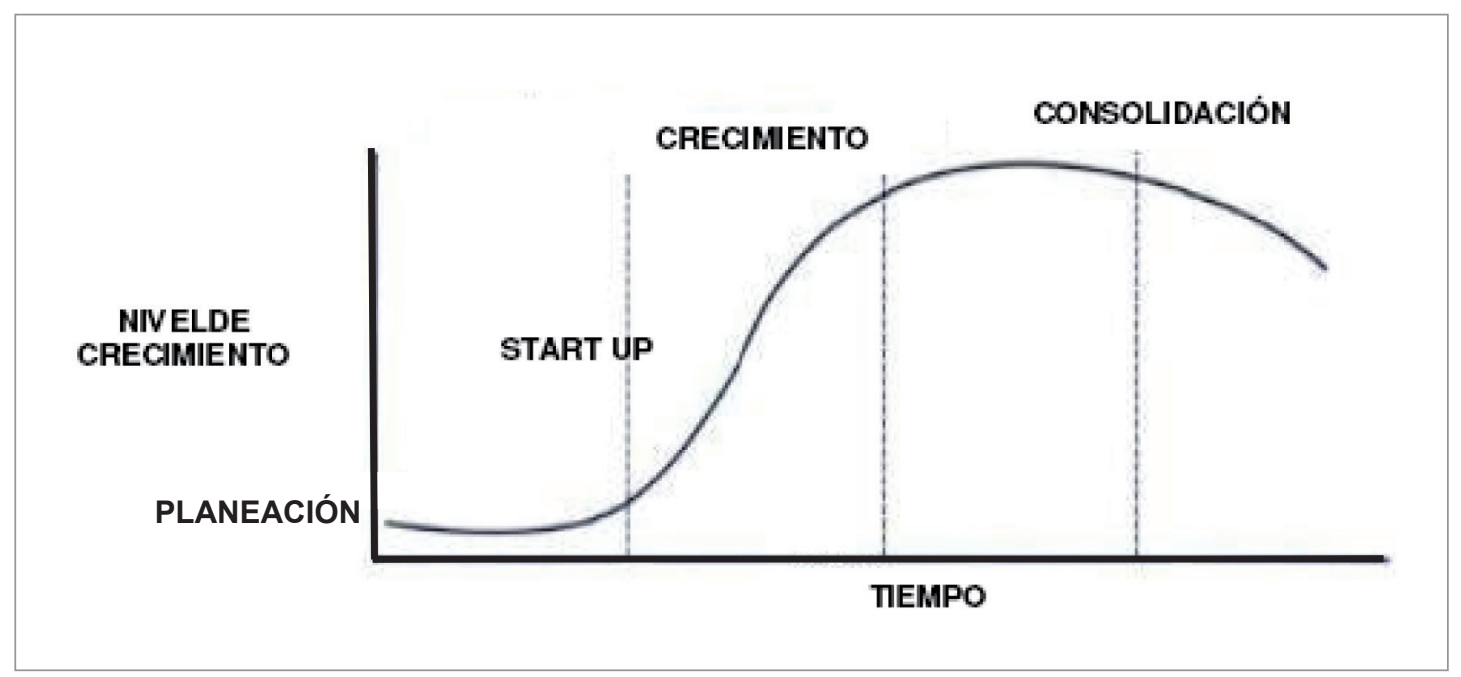

Fuente. Mogollón 2007 
financiamiento aportado por los fundadores y algunas fuentes cercanas a ellos. A medida que la empresa crece puede acceder a financiamiento a través de intermediarios, capital de riesgo y préstamos bancarios. Finalmente, aquellas empresas que sobreviven y continúan creciendo acceden a los mercados públicos.

El ciclo descrito por estos autores esta estrechamente relacionado a la "Pecking
Order Theory"5, que en los últimos años ha adquirido mayor fuerza ya que dicha teoría establece que las empresas no tienen una estructura de capital óptima a alcanzar, sino que siguen una escala de jerarquías a la hora de buscar financiación.

Lo que nos lleva a deducir que las etapas del desarrollo de vida de una empresa son totalmente incluyentes con la clase de financiación requerida por esta.

\section{LA CADENA DE FINANCIACIÓN EMPRESARIAL}

\section{I mirar la financiación como}

uno de los factores claves para el desarrollo empresarial, debido a su incidencia en las posibilidades de crecimiento de las unidades productivas, encontramos entonces que para cada una de las etapas anteriormente descritas existen diferentes modelos los cuales han sido diseñados de acuerdo con las condiciones y necesidades de la empresa y sus gestores; es así como surge la cadena de financiación empresarial como un ejercicio de articulación del portafolio financiero necesario por parte de los empresarios y sus organizaciones.

Para clasificar entonces las diferentes fuentes que conforman la cadena de financiación, es importante tener una visión a partir de las fuentes de los recursos, encontrando entonces tres grandes categorías tal y como se observa en el siguiente cuadro.

5 MYERS S. e MAJLUF, N. S. (1984), “ Corporate Financing and Investment Decisions when firms have information that investors do not have", Journal of Financial Economics, n 13, pp. 187-221. 


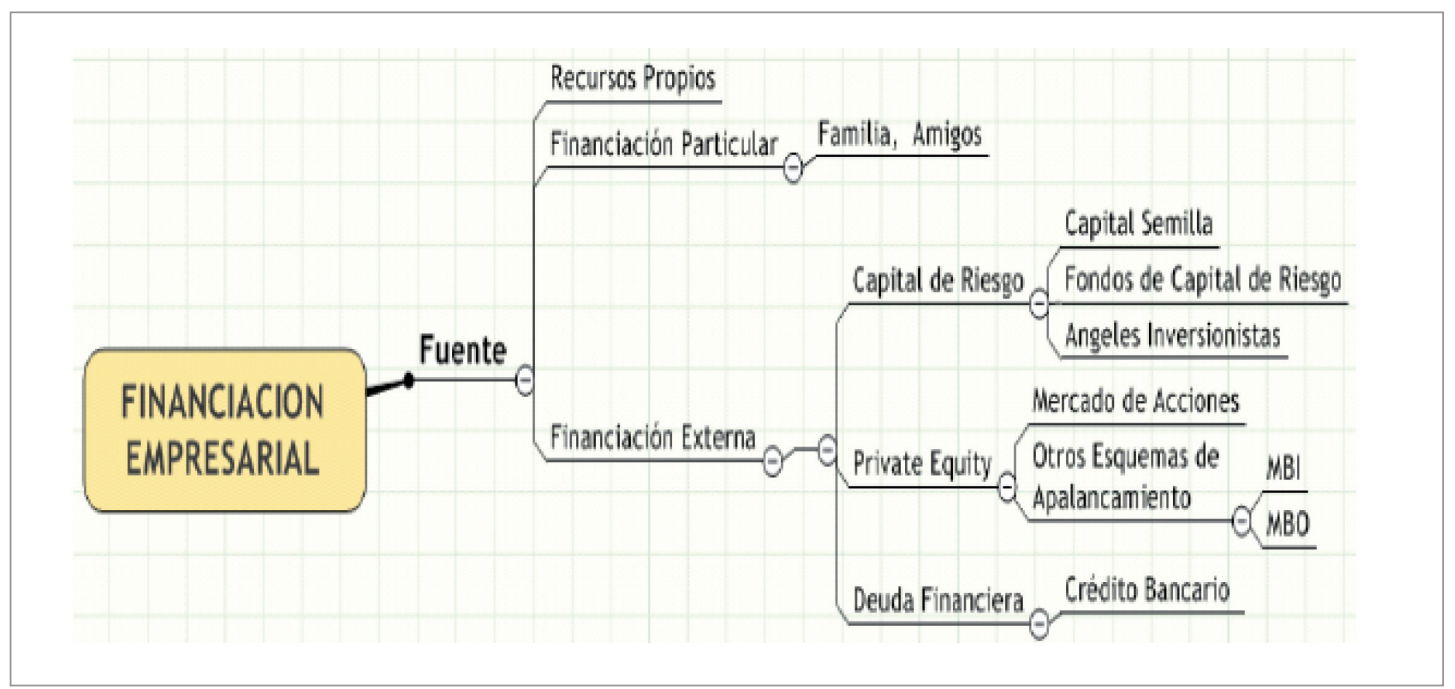

Fuente. Mogollón 2007

Es claro entonces que para las etapas de planeación o conceptualización, también denominada en algunos casos como semilla, es imprescindible el manejo de fuentes de recursos propios o de financiación particular, tal y como lo presentan para el caso Colombiano Castañeda y Fadul $(2002)^{6}$.

Sin embargo la verdadera problemática se presenta en el momento en que el emprendedor empresario debe conformarse con estas fuentes de financiación, sin poder acceder a nuevas y diferentes alternativas, que estén más acorde con sus necesidades y el nivel de desarrollo de su empresa, es allí donde se hace necesaria la conformación de una cadena de financiación que permita a las unidades productivas poder evolucionar y madurar sus modelos de negocio, contando con los recursos necesarios para tal fin, presentándose entonces un esquema de las siguientes características:

\begin{tabular}{|l|c|c|c|c|}
\hline \multicolumn{1}{c}{ Fuentes } & Semilla & Start Up & Desarrollo & Consolidación \\
\hline Buy Outs, Buy In & & & & $\square$ \\
\hline Bolsa & & & & $\square$ \\
\hline Bancos & & & $\square$ & $\square$ \\
\hline Fondos de capital de riesgo & $\square$ & $\square$ & $\square$ & $\square$ \\
\hline Inversionistas particulares & & $\square$ & $\square$ & \\
\hline Angeles de negocio & $\square$ & $\square$ & $\square$ & \\
\hline Recursos propios, amigos y familiares & $\square$ & $\square$ & & \\
\hline
\end{tabular}

Fuente. Mogollón 2007

6 Casañeda y Fadul (2002): "Globalización y crísis económica: incidencia en la financiación de unidades de pequeña escala". 


\subsection{Recursos propios (Bootstraping)}

Esta es la fuente más común de capital de financiamiento utilizada por los emprendedores de economías en desarrollo como es el caso particular de Colombia, y está compuesta por los fondos o ahorros propios o de sus familiares, los cuales utilizan para poner en marcha su proyecto empresarial; debido a las dificultades que presentan para acceder a fuentes de financiamiento convencionales.

En América Latina, el financiamiento temprano de las empresas que crecen rápidamente depende principalmente de los ahorros del emprendedor y del apoyo de familiares y amigos. $Y$ es América Latina quien presenta los niveles más altos de dependencia en relación con los ahorros internos. (Kantis y otros, 2004).

Los emprendedores que utilizan los recursos propios para el start up de su empresa siempre van a estar sujetos a desarrollar las actividades gerenciales y productivas en función de la minimización de costos para de esta forma poder cumplir con las actividades de la misma.

\subsection{Capital de riesgo (Venture capital)}

Las investigaciones acerca del mercado del Capital de Riesgo (CR) comenzaron en los Estados Unidos hacia los años 80 y se dio precisamente por el auge de las empresas de tecnología y la dificultad que estas tenían para encontrar financiamiento en el mercado de capital.

El Capital de Riesgo, tal y como se define en la Exposición de Motivos de la Ley 25/2005 reguladora de las entidades de capital riesgo y de sus sociedades gestoras, es una actividad financiera que consiste en proporcionar recursos a medio y largo plazo, pero sin vocación de permanencia ilimitada, a empresas que presentan dificultades para acceder a otras fuentes de financiación. Va dirigido a empresas no financieras y no cotizadas, durante su etapa de arranque (venture capital) o en su etapa de madurez, como consecuencia de un proceso de expansión o de reestructuración (private equity).

Actualmente existen diferentes tipos de inversores de riesgo, que han actuado en start-ups de la nueva economía y que han ayudado de una forma significativa a poner en marcha o dinamizar proyectos empresariales que difícilmente habrían podido acceder a otras fuentes de financiación estos son:

- Capital semilla(Seed Capita/). Modalidad de capital de riesgo que consiste en la financiación preliminar para el desarrollo de un producto o inversión.

7 LEY 25/2005 de24 de Noviembre; Reguladora de las entidades de capital de riesgo y sus sociedades gestoras-Jefatura del Estado (España)-JUAN CARLOS I REY DE ESPAÑA. 
- Capital de arranque (Start Up Capital) Modalidad de capital de riesgo dirigido a empresas ya iniciadas, necesitadas de recursos financieros para la iniciación de una actividad económica.
- Capital de expansión (Development capital). Aplicado al capital de riesgo, inversiones en empresas ya operativas que precisan ampliar líneas de producción, consolidar la compañía o recuperar el equilibrio financiero.

\section{3 Ángeles inversionistas (Informal venture capital)}

Los actores del mercado informal de capital de riesgo son conocidos en la literatura como Ángeles Inversores (Al). El término surgió en Estados Unidos a principios de 1900 para referirse a las personas que invertían dinero en las producciones teatrales de Broadway.

Los Ángeles Inversionistas, generalmente son empresarios exitosos o personas con un excedente suficiente de dinero para invertir en empresas, que están en las primeras etapas de formación.

Estos se diferencian de otro tipo de inversores porque además del aporte en capital financiero adicionan como valor agregado su asesoría, networking, y gestión, ya que por lo general un buen número de ellos tienen algún tipo de experiencia en el sector productivo o de servicios, lo cual utilizan para garantizar de una u otra forma el cumplimiento de sus expectativas de inversión.

Aunque varios autores se han dedicado ha desarrollar tipologías de angeles Inversores, en Colombia o en América Latina en donde esta figura es apenas conocida y este mercado incipiente, no figura o no existe una tipología que describa las clases de Ángeles existentes; Sin embargo se podría decir que estos asumen características según el país, su grado de desarrollo, la cultura emprendedora o la región en que se encuentran.

\subsection{Inversionistas privadas}

En países como México se han desarrollado figuras alternas a la de los Ángeles Inversionistas, tal es el caso de los Inversionistas Privados, quienes se diferencian de estos últimos en que su participación dentro de la empresa se limita a sus aportes en recursos financieros, es decir no se convierten en parte de la gestión y direccionamiento de la nueva empresa. 


\section{Tendencias a Nivel MUNdial}

s clara una tendencia hacia el desarrollo de esquemas de inversión de riesgo como soporte para el crecimiento económico y de la innovación, vía el apoyo a la creación de nuevas empresas, siendo estas en su mayoría de alto crecimiento o de base tecnológica. En el caso de los Estados Unidos, cuentan con instrumentos orientados a la creación y desarrollo de nuevas empresas desde mediados de los 60's. La Small Business Administration (SBA) y la National Commission on Entrepreneurship (NCOE), han sido las principales agencias públicas en donde se ha concentrado la asistencia técnica a la creación de nuevas empresas. Pero además han impulsado el financiamiento de la inversión de riesgo a través de las Small Business Investment Companies (SBICs) y de la financiación de la innovación a través del Small Business Innovation Research Program (SBIR).

Canadá fue el primero en adoptar una política deliberada de entrepreneurship a nivel nacional en 1988. Más recientemente, a mediados de la década pasada, países como España, los Países Bajos, Escocia, Taiwán, Suiza y Finlandia, entre otros, han destacado la importancia de las nuevas empresas en la generación de empleo estructural y el crecimiento económico. Esto ha llevado a plantear políticas orientadas a reducir las barreras a la creación de empresas, a reformar las leyes de quiebra, a impulsar una educación emprendedora en todos los niveles, y a mejorar la oferta de financiamiento para start-ups especialmente las tecnológicas y nuevas empresas en crecimiento ${ }^{8}$.

Es decir, el capital de riesgo en un mayor o menor grado está cubriendo de alguna forma el "financial gap", en los países desarrollados en los que como vemos se encuentra en un alto grado de evolución y existen reglas, políticas, normas y hasta leyes enfocadas a su mejor funcionamiento adaptándolo a condiciones propias de los mismos, y enfocándolo a cubrir verdaderas necesidades; como en el caso particular de España cuya ley 25/ de 2005 regula las entidades de capital de riesgo de este país.

Así mismo, en los países latinoamericanos en donde las asimetrías de información $y$ otros factores predominantes en los mecanismos financieros existentes, hacen compleja la accesibilidad a los mismos, el capital de riesgo está empezando a tomar un papel importante ayudando de esta manera a mejorar los procesos de creación de empresas, aunque hasta ahora esta fuente de financiamiento sea incipiente en economías tan poco desarrolladas como la Colombiana, hay ejemplos claros a destacar como el caso del Fondo Emprender.

Por otra parte, es evidente como distintos gobiernos se han propuesto apoyar el

\footnotetext{
${ }^{8}$ KANTIS, Hugo. Desarrollo emprendedor:América Latina y la experiencia internacional

9 PriceWaterhouse Coopers Venture Equity Latin AmericaLATAM - 2005.
} 
desarrollo de redes de inversores privados (business angels) como en el caso de Canadá, Escocia, Finlandia, Irlanda, el Reino Unido, EEUU y Taiwán o la realización de foros como en Estados Unidos, el Reino Unido o Japón para mejorar el flujo de información entre individuos con dinero para invertir y emprendedores que lo necesitan.

Es entonces claro que la industria de la financiación de nuevas empresas y específicamente la que se refiere a la inversión de riesgo, es un fenómeno aún muy nuevo, el cual hasta ahora no presenta cifras representativas en las economías en desarrollo como las latinoamericanas.

De acuerdo con un estudio realizado por la firma de consultoría PriceWaterhouse
Coopers $^{9}$, para el año 2003 la inversión en capital de riesgo alrededor del mundo alcanzó la cifra de US\$ 115.000 millones, valor equivalente al $0.4 \%$ del PIB mundial. Concentrándose la mayoría de estos esfuerzos en las regiones comprendidas por Europa y Norteamérica, quienes sumadas representan más del $80 \%$ del capital de riesgo a nivel mundial.

En el caso latinoamericano, donde de acuerdo con la información del mismo estudio se encuentra aproximadamente el $1 \%$ del capital de riesgo, los casos de mayor relevancia son los desarrollados en el Brasil, Argentina, México y Chile, siendo este último uno de los que presenta un desarrollo más reciente.

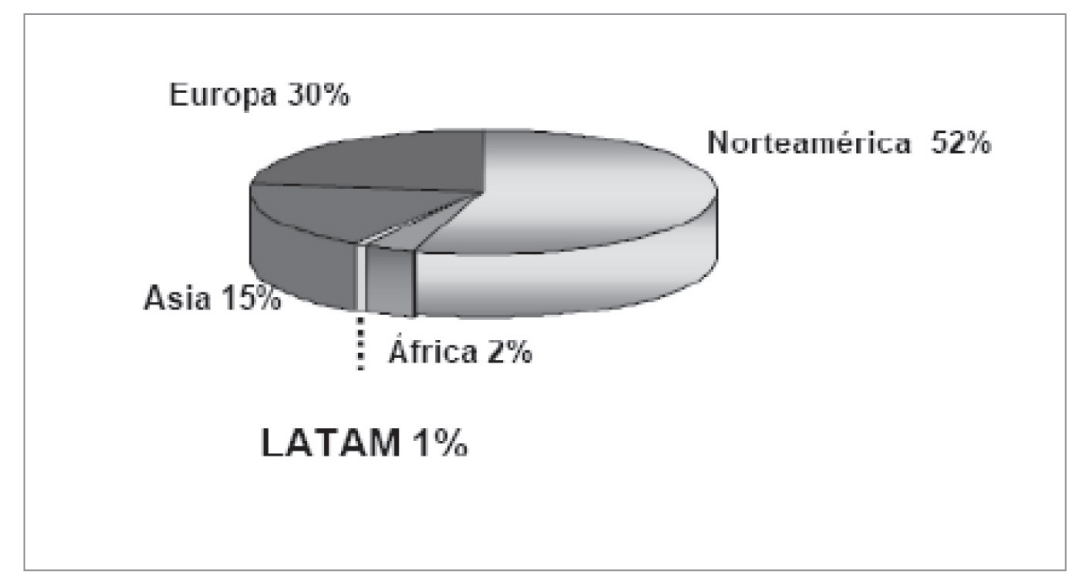

Fuente. Price Waterhouse Coopers Venture Equity Latin Ametica. 


\section{Conclusiones}

Es evidente la necesidad de generar nuevas alternativas para la financiación de las nuevas empresas en Colombia, el modelo actual en el que los emprendedores en su gran mayoría suplen sus necesidades financieras a través de los recursos propios o líneas de crédito no diseñadas para tal fin, llevan a que el desarrollo de las nuevas iniciativas empresariales pueda verse estancado por la falta de los volúmenes propicios de capital.

Lo anterior contrasta entonces con un clara tendencia mundial hacia la generación de lo que podríamos llamar una cultura de la inversión de riesgo, no solo en lo que respecta a fondos corporativos, o de aquellos interesados en la consolidación de pequeñas y medianas empresas; sino en muchos casos al apoyo de emprendimiento en sus primeras etapas de gestación, convirtiendo a las empresas en start up en una opción no solamente interesante desde el punto de vista de desarrollo económico y social, sino también significativa desde las posibilidades de generación de altos rendimientos para este tipo de inversionistas.

Ahora bien, es importante reflexionar un poco en los que se podrían determinar como los factores claves de éxito para la generación de esquemas de inversión de riesgo en un mercado hasta ahora incipiente en estos temas como lo es el colombiano; siendo algunos de ellos los siguientes:

- Es fundamental la articulación de los esfuerzos que entorno a la creación de empresas se generan en el país, en este caso es evidente como en las diferentes regiones un número significativo de entidades trabajan tanto en la sensibilización, formación y acompañamiento de emprendedores; como en el apoyo financiero y no financiero de las mismas, pero en la mayoría de los casos de manera aislada, evidenciándose este "divorcio" en mayor medida entre los representantes del sector académico (como lo pueden ser la Universidades) y el sector empresarial (los gremios empresariales).

- Es claro igualmente la necesidad de generar un verdadero mercado de capital de riesgo, en el que se asegure una demanda de recursos no solo representada por una gran cantidad de proyectos o emprendimientos, sino por propuestas realmente innovadoras y con alto potencial de crecimiento; pues como se pudo observar al analizar los ejemplos tanto europeos como norteamericanos, este es uno de los pilares sobre los que se soportan las acciones de las redes de ángeles inversionistas, siendo esta una condición obvia pues solo este tipo de iniciativas pueden llegar a ofrecer a los inversores las posibilidades de acceder a altas tasas de rentabilidad en el mediano plazo.

En el caso colombiano, las iniciativas frente al tema aún son mínimas, sin embargo las condiciones macroeconómicas presentan posibilidades interesantes a las inversiones en general, lo anterior así como el evidente interés por los gobiernos tanto central como regionales en el apoyo a proyectos 
y políticas encaminadas a la creación de nuevas empresas, representan entonces las condiciones necesarias para desarrollar este tipo de alternativas.

Tal como se evidencia en el caso europeo, la consolidación de un cultura de la inversión encaminada a las nueves empresas, debe entonces soportarse principalmente en la generación de confianza y reconocimiento entre las partes, es fundamental identificar a los posibles inversores para sensibilizarlos frente a su papel como ángeles y los beneficios tanto económicos como sociales que este papel les puede implicar, así como a los emprendedores de aquellos proyectos y empresas que cuenten con las características necesarias para poder hacer parte de este tipo de procesos.

\section{Bibliografía}

Alternativa a la Financiación de la Pyme.(2005) - Ministerio de Industria, Turismo y Comercio (España)

Benjamin, G. A. \& Margulis J. (2000): Angel financing: How to find and invest in private.

Berger, Allen N. y Udell, Gregory F. (1998). The Economics of Small Business Finance: The Roles of Private Equity and Debt Markets in the Financial Growth Cycle. Journal of Banking and Finance. Volumen 22.

Business Angel Investing Groups Growing in North America.(2002) - Kauffman Foundation October.

CONPES 3484. Política nacional para la transformación productiva y la promoción de las micro, pequeñas y medianas empresas: un esfuerzo público-privado-Ministerio de Comercio Industria y Turismo, Departamento Nacional de Planeación - Agosto de 2007.

Coveney, P. and Moore, K. (1998): Business Angels: Securing Start- Up Finance. John Wiley \& Son, London, UK.

Downes John Y Elliot J,(2002): Diccionario de términos de finanzas e inversiones equity. John Wiley \& Son, New York, USA.

Echecopar Germán.(2005): Capital semilla para el financiamiento de las nuevas empresas. Avances y lecciones aprendidas en América Latina - Banco Interamericano de Desarrollo. 
Fama, E. F. y French, K. R. (2002): "Testing trade-off and pecking order predictions about dividends and debt". The Review of Financial Studies, vol. 15.

Global Entrepreneurship Monitor. Informe Colombia 2006.

Informe 35 ${ }^{\mathrm{a}}$ Asamblea General de ALIDE - 2005.

Kantis Hugo et al,(2004): Desarrollo emprendedor: América Latina y la experiencia internacional.

Ley 25/2005 de 24 de noviembre. Reguladora de las entidades de capital de riesgo y sus sociedades gestoras-Jefatura del Estado (España)-Juan Carlos I Rey de España.

Los Fondos de capital de riesgo como alternativa de financiamiento de las empresas colombianas (2002)- Asobancaria.

Mogollón Cuevas Yida (2207): Fuentes de financiación para el Start Up de una empresa.

Raposo Mario.(2005): Seminario promoción de la actividad emprendedora y apoyo a la creación de empresas - Universidad e da Beira Interior. 